\title{
A fully discrete $\varepsilon$-uniform method for singular perturbation problems on equidistant meshes
}

\author{
Ali Filiz , Ali Nesliturk \& Ali Sendur
}

To cite this article: Ali Filiz , Ali Nesliturk \& Ali Sendur (2012) A fully discrete $\varepsilon$-uniform method for singular perturbation problems on equidistant meshes, International Journal of Computer Mathematics, 89:2, 190-199, DOI: 10.1080/00207160.2011.632411

To link to this article: http://dx.doi.org/10.1080/00207160.2011.632411

曲 Published online: 07 Nov 2011.

Submit your article to this journal $\sqsubset$

Џ Article views: 124

View related articles $\square$

Citing articles: 1 View citing articles 5 


\title{
A fully discrete $\epsilon$-uniform method for singular perturbation problems on equidistant meshes
}

\author{
Ali Filiz ${ }^{\mathrm{a}}$, Ali Nesliturk ${ }^{\mathrm{b} *}$ and Ali Sendur ${ }^{\mathrm{b}}$ \\ ${ }^{a}$ Department of Mathematics, Adnan Menderes University, 09010 Aydin, Turkey; ${ }^{b}$ Department of \\ Mathematics, Izmir Institute of Technology, 35430 Izmir, Turkey
}

(Received 17 November 2010; resubmitted 6 January 2011; revised revision received 22 February 2011; second revision received 5 April 2011; third revision received 11 July 2011; fourth revision received 13 September 2011; accepted 11

October 2011)

\begin{abstract}
We propose a fully discrete $\epsilon$-uniform finite-difference method on an equidistant mesh for a singularly perturbed two-point boundary-value problem (BVP). We start with a fitted operator method reflecting the singular perturbation nature of the problem through a local BVP. However, to solve the local BVP, we employ an upwind method on a Shishkin mesh in local domain, instead of solving it exactly. Thus, we show that it is possible to develop a $\epsilon$-uniform method, totally in the context of finite differences, without solving any differential equation exactly. We further study the convergence properties of the numerical method proposed and prove that it nodally converges to the true solution for any $\epsilon$. Finally, a set of numerical experiments is carried out to validate the theoretical results computationally.
\end{abstract}

Keywords: finite differences; uniform convergence; singular perturbation; fitted operator method; Shishkin mesh

2010 AMS Subject Classifications: 65L10; 65L11; 65L12

\section{Introduction}

It is well known that the classical finite-difference methods for the approximation of singularly perturbed boundary-value problems (BVPs) do not work in the critical range of $\epsilon$ where $\epsilon$ is considerably small compared with the mesh parameter $h$. Although the centred difference approximation produces good approximations for large values of $\epsilon$, the result is totally unphysical as $\epsilon \rightarrow 0$. These deficiencies disappear if we discretize the convection term by an appropriate one-sided finite-difference operator, in which case the resulting numerical method is known as the upwind method. However, the approximate solution may not uniformly converge to the true solution in the layer region where the useful information is confined. Therefore, it is important to devise uniformly convergent methods that yield the numerical approximations consistent with the physical configuration of the problem in all regimes.

\footnotetext{
*Corresponding author. Email: alinesliturk@iyte.edu.tr

ISSN 0020-7160 print/ISSN 1029-0265 online

(C) 2012 Taylor \& Francis

http://dx.doi.org/10.1080/00207160.2011.632411

http://www.tandfonline.com
} 
A considerable amount of research works have been devoted to the development of the uniformly convergent methods. In the construction of $\epsilon$-uniform finite-difference methods, two major approaches have generally been taken to date. The first of these involves replacing the standard finite-difference operator by a difference operator which reflects the singularly perturbed nature of the differential operator. Such numerical methods are referred to, in general, as fitted operator finite-difference methods $[4,5]$. Typical derivation of such methods based on the discretization of the domain into a set of equidistant subintervals and the exact solution of a local BVP with an irregular data on a pair of adjacent subintervals. It is appreciated that the method uses an equidistant mesh but the overall method suffers from the fact that it depends on the exact solution which is not easier to solve than the original problem.

The second major approach in the construction of the $\epsilon$-uniform finite-difference method involves the use of a fitted mesh; a mesh that is adapted according to the singular perturbation [4,5]. Let us concentrate on the most efficient subclass of the fitted meshes methods: Shishkin mesh [6]. A Shishkin mesh, also called a piecewise uniform fitted mesh, consists of a union of finite number of uniform meshes having different mesh parameters on both sides of a transition point. It turns out that a Shishkin mesh together with the simple upwind method is sufficient for the construction of an $\epsilon$-uniform method [7]. These meshes can also be applied to singular perturbation problems with interior layers caused by point sources [3]. The simplicity of Shishkin mesh is due to the use of equidistant subintervals on both sides of a transition point and this property is considered to be one of its major attractions. However, it requires the precise location of the layer structure a priori.

The algorithm investigated in this work combines these two major classes of $\epsilon$-uniform finitedifference methods. We start with a fitted operator method reflecting the singular perturbation nature of the problem through a local BVP posed on an adjacent pair of subintervals. However, the local BVP has an interior layer caused by a concentrated source and instead of solving it exactly, we approximate it with the upwind method on a Shishkin-like mesh on the patch of these subintervals. The distribution of the mesh points in the subdomain is determined depending on the local flow regime. Further, we prove that the resulting numerical method nodally converges to the true solution for any $\epsilon$. Thus, we display that it is possible to develop an $\epsilon$-uniform finitedifference method on equidistant meshes without solving the local differential equation exactly. This fact is also confirmed by numerical experiments presented below.

The layout of the paper is as follows. We present the numerical method proposed in Section 2 and investigate its convergence properties in Section 3. Therein, we also prove that the new algorithm nodally converges to the true solution uniformly. Finally, we perform the numerical tests in Section 4.

\section{Numerical method}

Consider the following singularly perturbed BVP on the unit interval $\Omega=(0,1)$

$$
\begin{aligned}
& \text { Find } u(x) \text { such that } \quad u(0)=u_{0}, \quad u(1)=u_{1} \quad \text { and } \\
& L u=-\epsilon u^{\prime \prime}+b(x) u^{\prime}+c(x) u=f(x) \quad \forall x \in \Omega
\end{aligned}
$$

under the assumptions that $0<\epsilon \leq 1, b(x) \geq b_{0}>0$ and $c(x) \geq 0$, where $u_{0}$ and $u_{1}$ are given constants. Define a uniform mesh $\left\{x_{i}\right\}_{i=0}^{N}$, where $x_{i}=i h, i=0,1, \ldots, N$ and $h=1 / N$, denoted by $\Omega^{N}$, the space of all mesh functions defined on $\Omega^{N}$ by $V\left(\Omega^{N}\right)$ and the discrete maximum norm for any mesh function $V$ by $\|V\|_{\Omega^{N}}=\max _{0 \leq i \leq N}\left|V_{i}\right|$. We set $b_{i}=b\left(x_{i}\right), c_{i}=c\left(x_{i}\right)$ and $f_{i}=f\left(x_{i}\right)$ throughout the paper. Let us try to solve the problem (1) by an $\epsilon$-uniform difference method of fitted operator type [4,5] on the uniform mesh $\Omega^{N}$. Typical derivation of the fitted operator methods 


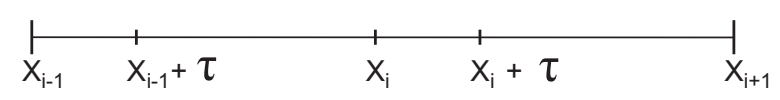

Figure 1. Subdomains of $\bar{\Omega}_{i} \cup \bar{\Omega}_{i+1}$.

is based on the exact solution of a local BVP reflecting the singular nature of the problem. The explicit statement of the local problem reads: Find the local function $g_{i}$, defined with respect to the mesh point $x_{i}$, on $\bar{\Omega}_{i} \cup \bar{\Omega}_{i+1}$ such that

$$
\begin{aligned}
M^{*} g_{i} & =-\epsilon g_{i}^{\prime \prime}(x)-b_{i} g_{i}^{\prime}(x)=\delta_{x_{i}}(x) \quad \forall x \in \Omega_{i} \cup \Omega_{i+1}, \\
g_{i}\left(x_{i-1}\right) & =0, \\
g_{i}\left(x_{i+1}\right) & =0,
\end{aligned}
$$

where $\Omega_{i}=\left(x_{i-1}, x_{i}\right)$. Equation (2) should be read in the sense of distributions. Multiplying the equation $L u=f$ with $g_{i}$, integrating the resulting expression from $x_{i-1}$ to $x_{i+1}$ and using the integration by parts and the continuity of $u$, respectively, we get the following identity:

$$
-\epsilon g_{i}^{\prime}\left(x_{i-1}\right) U_{i-1}+U_{i}+\epsilon g_{i}^{\prime}\left(x_{i+1}\right) U_{i+1}=\left(f_{i}-c_{i} U_{i}\right) \int_{x_{i-1}}^{x_{i+1}} g_{i} \mathrm{~d} x .
$$

However, the evaluation of $g_{i}^{\prime}\left(x_{i-1}\right)$ and $g_{i}^{\prime}\left(x_{i+1}\right)$ requires the exact solution of Equation (2) which may be difficult as much as the original problem (1). Therefore, we approximate the local Green function $g_{i}$ by a fitted mesh method of Shishkin type [4,5,7] and then use the resulting approximations in place of $g_{i}$ 's in Equation (3).

In that context, we formulate a fitted mesh method of Shishkin type on the union of $\Omega_{i}$ and $\Omega_{i+1}$ : divide $\bar{\Omega}_{i} \cup \bar{\Omega}_{i+1}$ into the four subintervals $\left[x_{i-1}, x_{i-1}+\tau\right],\left[x_{i-1}+\tau, x_{i}\right],\left[x_{i}, x_{i}+\tau\right]$ and $\left[x_{i}+\tau, x_{i+1}\right]$ (Figure 1) each has $M / 4$ mesh elements, where $\tau=\min \left\{h / 2, \epsilon / b_{i} \ln M\right\}$. The corresponding mesh parameters becomes $h_{1}^{*}=4 \tau / M$ and $h_{2}^{*}=(4 / M)(h-\tau)$. Thus, the Shishkin fitted mesh $\Omega_{i, \tau}^{M / 2} \cup \Omega_{i+1, \tau}^{M / 2}=\left\{x_{j}^{*}\right\}_{j=0}^{M}$ is defined by

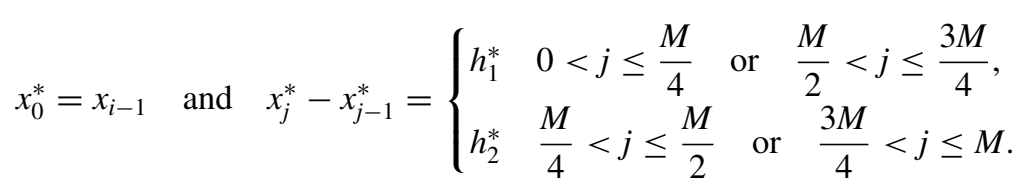

The discrete problem for Equation (2), using the upwind difference operator on the specified mesh (4), is given by

$$
\begin{aligned}
& \text { Find } G \in V\left(\Omega_{i, \tau}^{M / 2} \cup \Omega_{i+1, \tau}^{M / 2}\right) \text { such that } G_{0}=0, \quad G_{M}=0 \quad \text { and } \\
& -\epsilon D_{*}^{+} D_{*}^{-} G_{j}-b_{i} D_{*}^{+} G_{j}=\Delta_{x_{i}, j}, \quad 1 \leq j \leq M-1,
\end{aligned}
$$

where we mean $G_{j}^{i}$ by $G_{j}$ with $G_{j} \approx g_{i}\left(x_{j}^{*}\right)$, and

$$
D_{*}^{+} v_{j}=\frac{v_{j+1}-v_{j}}{h_{j+1}^{*}}, \quad D_{*}^{-} v_{j}=\frac{v_{j}-v_{j-1}}{h_{j}^{*}} \quad \text { and } \quad \Delta_{x_{i}, j}= \begin{cases}\frac{1}{h_{j+1}^{*}} & x_{i} \in\left[x_{j}^{*}, x_{j+1}^{*}\right), \\ 0 & \text { otherwise. }\end{cases}
$$

To solve Equation (5), we start with combining terms with the same indices together and obtain a three-point difference scheme:

$$
\left(-\lambda_{j}^{*}\right) G_{j+1}+\left(\frac{h_{j+1}^{*}}{h_{j}^{*}}+\lambda_{j}^{*}\right) G_{j}+\left(-\frac{h_{j+1}^{*}}{h_{j}^{*}}\right) G_{j-1}=\Delta_{x_{i}, j}, \quad j=1,2, \ldots, M-1,
$$


where $\lambda_{j}^{*}$ is defined by

$$
\lambda_{j}^{*}=\left\{\begin{array}{l}
\lambda_{1} \quad 1 \leq j \leq \frac{M}{4} \quad \text { or } \quad \frac{M}{2}<j \leq \frac{3 M}{4}, \\
\lambda_{2} \quad \frac{M}{4}<j \leq \frac{M}{2}
\end{array} \text { or } \quad \frac{3 M}{4}<j \leq M-1, \quad \text { with } \lambda_{1}=1+\frac{b_{i} h_{1}^{*}}{\epsilon}, \quad \lambda_{2}=1+\frac{b_{i} h_{2}^{*}}{\epsilon} .\right.
$$

At the interior points of the subintervals, the difference equation (6) takes the simple form

$$
\left(-\lambda_{j}^{*}\right) G_{j+1}+\left(1+\lambda_{j}^{*}\right) G_{j}+(-1) G_{j-1}=0
$$

for which, the roots of the characteristic polynomial are $r_{1}=1$ and $r_{2}=1 / \lambda_{j}^{*}$. Therefore, we may assume that the solution of the difference equation (5) is of the form

$$
G_{j}^{i}= \begin{cases}a_{1}+a_{2} \lambda_{1}^{-j} & \text { if } 0 \leq j \leq \frac{M}{4}, \\ a_{3}+a_{4} \lambda_{2}^{-j} & \text { if } \frac{M}{4} \leq j \leq \frac{M}{2}, \\ a_{5}+a_{6} \lambda_{1}^{-j} & \text { if } \frac{M}{2} \leq j \leq \frac{3 M}{4}, \\ a_{7}+a_{8} \lambda_{2}^{-j} & \text { if } \frac{3 M}{4} \leq j \leq M .\end{cases}
$$

Now, we need to determine the coefficients $a_{k}, k=1, \ldots, 8$, in order to obtain the solution of the difference equation explicitly. The boundary conditions $G_{0}=G_{M}=0$ give us two equations. Three equations come from the difference equations (6) written at the transition points $x_{M / 4}^{*}, x_{M / 2}^{*}$ and $x_{3 M / 4}^{*}$, respectively. The other three equations are obtained by imposing the continuity of the difference solutions at transition points

$$
\begin{gathered}
a_{1}+a_{2} \lambda_{1}^{-M / 4}=a_{3}+a_{4} \lambda_{2}^{-M / 4}, \\
a_{3}+a_{4} \lambda_{2}^{-M / 2}=a_{5}+a_{6} \lambda_{1}^{-M / 2}, \\
a_{5}+a_{6} \lambda_{1}^{-3 M / 4}=a_{7}+a_{8} \lambda_{2}^{-3 M / 4} .
\end{gathered}
$$

Once we solve these eight equations for $a_{i}, i=1, \ldots, 8$, we substitute them into Equation (8) to get the solution of the difference equation (5) in an explicit manner

$$
G_{j}^{i}= \begin{cases}\frac{h_{2}^{*}}{\eta} \kappa_{1} \kappa_{2} \kappa_{3}\left(1-\lambda_{1}^{-j}\right) & \text { if } 0 \leq j \leq \frac{M}{4}, \\ \frac{h_{2}^{*}}{\eta}\left(\kappa_{1} \kappa_{2} \kappa_{3}+\kappa_{2}-\kappa_{2} \kappa_{3}-\lambda_{2}^{-j+M / 2}\right) & \text { if } \frac{M}{4}<j \leq \frac{M}{2}, \\ \frac{h_{2}^{*}}{\eta}\left(\lambda_{2} \kappa_{2}-\lambda_{1} \kappa_{2}-\lambda_{2}+\kappa_{2} \kappa_{3} \lambda_{1}^{-j+3 M / 4}\right) & \text { if } \frac{M}{2}<j \leq \frac{3 M}{4}, \\ \frac{h_{2}^{*}}{\eta}\left(-1+\lambda_{2}^{-j+M}\right) & \text { if } \frac{3 M}{4}<j \leq M,\end{cases}
$$

where $\kappa_{1}=\lambda_{1}^{M / 4}, \kappa_{2}=\lambda_{2}^{M / 4}, \kappa_{3}=\lambda_{1} \lambda_{2}^{-1}$ and $\eta=\epsilon\left(\lambda_{1}-1\right)\left(1+\lambda_{1}^{M / 4} \lambda_{2}^{M / 4}\right)$. Now, we replace $g_{i}^{\prime}\left(x_{i-1}\right)$ and $g_{i}^{\prime}\left(x_{i+1}\right)$ in Equation (3) by using the discrete Green function $G^{i}$ in their one-sided 
approximations, that is

$$
g_{i}^{\prime}\left(x_{i-1}\right) \approx D^{+} G_{0}=\frac{G_{1}-G_{0}}{h_{1}^{*}}, \quad g_{i}^{\prime}\left(x_{i+1}\right) \approx D^{-} G_{M}=\frac{G_{M}-G_{M-1}}{h_{2}^{*}},
$$

which yields the ultimate numerical method that

$$
-\epsilon D^{+} G_{0} \tilde{U}_{i-1}+\tilde{U}_{i}+\epsilon D^{-} G_{M} \tilde{U}_{i+1}=\left(f_{i}-c_{i} \tilde{U}_{i}\right) \int_{x_{i-1}}^{x_{i+1}} G^{i} \mathrm{~d} x .
$$

The method (10) is remarkable in the sense that it requires no exact solution of Equation (2) at all. In the implementation stage, we may directly use the values of the approximate Green function $G^{i}$ from Equation (5). Thus, from the implementation point of view, we do not even need to find the explicit expressions for $G^{i}$ in Equation (9), as they are only needed to prove that the method (10) is $\epsilon$-uniform convergent in Section 3.

\section{Convergence properties}

To investigate the convergence properties of the numerical method (10), we should recall some known results that are needed to prove that the method under consideration converges uniformly in $\epsilon$. Let us first recall the exact scheme (also known as Il'in-Allen-Southwell method) obtained by the fitted operator method for the problem (1) in the upwind form $[2,5]$ :

$$
\begin{aligned}
& \text { Find } U \in V\left(\Omega^{N}\right) \quad \text { such that } U_{0}=u_{0}, \quad U_{N}=u_{1} \quad \text { and } \\
& \quad-\epsilon B\left(\rho_{i}\right) D^{+} D^{-} U_{i}+b_{i} D^{-} U_{i}+c_{i} U_{i}=f_{i}, \quad 1 \leq i \leq N-1,
\end{aligned}
$$

where $B\left(\rho_{i}\right)=\rho_{i} /\left(e^{\rho_{i}}-1\right)$ is Bernoulli's function. On the other hand, let us rewrite the numerical method (10) in the upwind form of

$$
-\epsilon \sigma_{i} D^{+} D^{-} \tilde{U}_{i}+\eta_{i} b_{i} D^{-} \tilde{U}_{i}+\theta_{i} c_{i} \tilde{U}_{i}=f_{i},
$$

where

$$
\sigma_{i}=\frac{h^{2}}{\epsilon} \frac{T_{2}}{T_{3}}, \quad \eta_{i}=\frac{h^{2}}{\epsilon \rho_{i}} \frac{T_{1}-T_{2}}{T_{3}}, \quad \theta_{i}=\frac{1}{c} \frac{1+c T_{3}-T_{1}-T_{2}}{T_{3}}
$$

and

$$
T_{1}\left(\epsilon, b_{i}, h, M\right)=\epsilon D^{+} G_{0}, \quad T_{2}\left(\epsilon, b_{i}, h, M\right)=-\epsilon D^{-} G_{M}, \quad T_{3}\left(\epsilon, b_{i}, h, M\right)=\int_{x_{i-1}}^{x_{i+1}} G^{i} \mathrm{~d} x .
$$

It is well known that the schemes of upwind type whose coefficients are close to the coefficients of the method (11) are also uniformly convergent [1]. We will use this fact to show that the method (10) is uniformly convergent, by proving that the coefficients $\sigma_{i}, \eta_{i}$ and $\theta_{i}$ in Equation (12) can be made arbitrarily close to the coefficients of the exact scheme (11). Thus, we need to prove that

$$
\begin{aligned}
& \lim _{M \rightarrow \infty} \sigma_{i}\left(\epsilon, b_{i}, h, M\right)=B\left(\rho_{i}\right), \\
& \lim _{M \rightarrow \infty} \eta_{i}\left(\epsilon, b_{i}, h, M\right)=1, \\
& \lim _{M \rightarrow \infty} \theta_{i}\left(\epsilon, b_{i}, h, M\right)=1 .
\end{aligned}
$$

Since $G^{i}$ is a strictly positive function, so does the number $T_{3}$. Therefore, we can first evaluate $\lim _{M \rightarrow \infty} T_{i}$ for $i=1,2,3$ in Equation (14), and then use the relations in Equation (13) to find the limits (15)-(17). 
Lemma 3.1 Let $T_{1}\left(\epsilon, b_{i}, h, M\right)$ be given as in Equation (14), that is, $T_{1}\left(\epsilon, b_{i}, h, M\right)=$ $\epsilon\left(G_{1}-G_{0}\right) / h_{1}^{*}$. If $\rho_{i}=b_{i} h / \epsilon$ is fixed, then we have

$$
\lim _{M \rightarrow \infty} T_{1}\left(\epsilon, b_{i}, h, M\right)=\frac{e^{\rho_{i}}}{1+e^{\rho_{i}}} .
$$

Proof Set $\rho=\rho_{i}$ and $b=b_{i}$. We need to consider two cases with respect to the transition parameter $\tau$. In the first case, where $\tau=h / 2$, the mesh is uniform with $h_{1}^{*}=h_{2}^{*}=2 h / M$ and $\lambda_{1}=\lambda_{2}=1+2 b h /(M \epsilon)$. Using these parameter values, we rewrite $T_{1}$ by rearranging the terms and using the explicit solution of $G^{i}$ in Equation (9)

$$
\begin{aligned}
\frac{G_{1}-G_{0}}{h_{1}^{*}} & =\frac{1}{h_{1}^{*}} \frac{h_{2}^{*}}{\epsilon} \frac{\lambda_{1}^{M / 4+1} \lambda_{2}^{M / 4-1}\left(1-\lambda_{1}^{-1}\right)}{\left(\lambda_{1}-1\right)\left(1+\lambda_{1}^{M / 4} \lambda_{2}^{M / 4}\right)} \\
& =\frac{1}{\epsilon} \frac{\lambda_{1}^{M / 4} \lambda_{2}^{M / 4}}{\lambda_{2}\left(1+\lambda_{1}^{M / 4} \lambda_{2}^{M / 4}\right)} \\
& =\frac{1}{\epsilon} \frac{(1+2 b h /(M \epsilon))^{M / 4}(1+2 b h /(M \epsilon))^{M / 4}}{(1+2 b h /(M \epsilon))\left[1+(1+2 b h /(M \epsilon))^{M / 4}(1+2 b h /(M \epsilon))^{M / 4}\right]} \\
& =\frac{1}{\epsilon} \frac{(1+2 \rho / M)^{M / 2}}{(1+2 \rho / M)\left[1+(1+2 \rho / M)^{M / 2}\right]} .
\end{aligned}
$$

Using the fact that $\lim _{M \rightarrow \infty}(1+x / M)^{M}=e^{x}$ for any $x \in \mathbb{R}$, the last expression immediately leads to

$$
\lim _{M \rightarrow \infty} \frac{G_{1}-G_{0}}{h_{1}^{*}}=\frac{1}{\epsilon} \frac{e^{\rho}}{1+e^{\rho}} .
$$

In the second case, where $\tau=\epsilon / b \ln M$, the mesh is piecewise uniform with the mesh parameters $h_{1}^{*}=(4 / M)(\epsilon / b) \ln M / 2$ and $h_{2}^{*}=(4 / M)(h-\epsilon / b \ln M / 2)$, in which case, $\lambda_{1}=$ $1+4 / M \ln M / 2$ and $\lambda_{2}=1+(4 \rho / M)-4 / M \ln M / 2$. Now,

$$
\begin{aligned}
& \frac{G_{1}-G_{0}}{h_{1}^{*}}=\frac{1}{\epsilon} \frac{h_{2}^{*}}{h_{1}^{*}} \frac{\lambda_{1}^{M / 4} \lambda_{2}^{M / 4}}{\lambda_{2}\left(1+\lambda_{1}^{M / 4} \lambda_{2}^{M / 4}\right)} \\
&=\frac{1}{\epsilon} \frac{h_{2}^{*}}{h_{1}^{*}} \frac{\left(1+b h_{1} / \epsilon\right)^{M / 4}\left(1+b h_{2} / \epsilon\right)^{M / 4}}{\left(1+b h_{2} / \epsilon\right)\left[1+\left(1+b h_{1} / \epsilon\right)^{M / 4}\left(1+b h_{2} / \epsilon\right)^{M / 4}\right]} \\
&= \frac{1}{\epsilon}\left(\frac{\rho}{\ln M / 2}-1\right) \frac{\left(1+(4 \rho / M)-16 \ln M / 2 / M^{2}(\ln M / 2-\rho)\right)^{M / 4}}{(1+(4 \rho / M)-4 \ln M / M)(1+(1+(4 \rho / M)} . \\
&\left.\left.-16 \ln M / 2 / M^{2}(\ln M / 2-\rho)\right)^{M / 4}\right)
\end{aligned} .
$$

Taking again the limit of the last expression as $M \rightarrow \infty$ gives us the desired result

$$
\lim _{M \rightarrow \infty} \frac{G_{1}-G_{0}}{h_{1}^{*}}=\frac{1}{\epsilon} \frac{e^{\rho}}{1+e^{\rho}} .
$$

Lemma 3.2 Let $T_{2}\left(\epsilon, b_{i}, h, M\right)$ be given as in Equation (14), that is, $T_{2}\left(\epsilon, b_{i}, h, M\right)=$ $-\epsilon\left(G_{M}-G_{M-1}\right) / h_{2}^{*}$. If $\rho_{i}=b_{i} h / \epsilon$ is fixed, then we have

$$
\lim _{M \rightarrow \infty} T_{2}\left(\epsilon, b_{i}, h, M\right)=\frac{1}{1+e^{\rho_{i}}} .
$$


Proof We use the difference solution $G^{i}$ in Equation (9) and the same arguments employed in the proof of Lemma 3.1. Set $\rho=\rho_{i}$ and $b=b_{i}$. In the uniform case where $\tau=h / 2$, with $h_{1}^{*}=h_{2}^{*}=2 h / M$ and we have

$$
\frac{G_{M}-G_{M-1}}{h_{2}^{*}}=-\frac{1}{\epsilon} \frac{h_{2}^{*}}{h_{1}^{*}} \frac{1}{\left(1+\lambda_{1}^{M / 4} \lambda_{2}^{M / 4}\right)}=-\frac{1}{\epsilon} \frac{1}{\left[1+\left(1+\frac{2 \rho}{M}\right)^{M / 2}\right]}
$$

which immediately yields

$$
\lim _{M \rightarrow \infty} \frac{G_{M}-G_{M-1}}{h_{2}^{*}}=-\frac{1}{\epsilon} \frac{1}{1+e^{\rho}} .
$$

For the other case where $\tau=\epsilon / b \ln M$, a calculation shows that

$$
\frac{G_{M}-G_{M-1}}{h_{2}^{*}}=-\frac{1}{\epsilon} \frac{h_{2}^{*}}{h_{1}^{*}} \frac{1}{\left(1+\lambda_{1}^{M / 4} \lambda_{2}^{M / 4}\right)}=\frac{1}{\epsilon} \frac{(\rho / \ln M / 2)-1}{1+(1+(4 \ln M / 2) / M)^{M / 4}(1+(4 \rho / M)}
$$

from which we easily get

$$
\lim _{M \rightarrow \infty} \frac{G_{M}-G_{M-1}}{h_{2}^{*}}=-\frac{1}{\epsilon} \frac{1}{1+e^{\rho}} .
$$

Lemma 3.3 Let $T_{3}\left(\epsilon, b_{i}, h, M\right)$ be given as in Equation (14), that is,

$$
T_{3}\left(\epsilon, b_{i}, h, M\right)=\int_{x_{i-1}}^{x_{i+1}} G^{i} \mathrm{~d} x .
$$

If $\rho_{i}=b_{i} h / \epsilon$ is fixed, then we have

$$
\lim _{M \rightarrow \infty} T_{3}\left(\epsilon, b_{i}, h, M\right)=\frac{h}{b_{i}} \frac{e^{\rho_{i}}-1}{e^{\rho_{i}}+1} .
$$

Proof We give the proof for the uniform case only. For the non-uniform case, the steps are longer but similar, so we omit it. Let us use the explicit solution of $G^{i}$ in Equation (9) and the composite trapezium quadrature rule to evaluate Equation (20):

$$
\begin{aligned}
\int_{x_{i-1}}^{x_{i+1}} G^{i} \mathrm{~d} x= & \left(\frac { 1 } { ( b ^ { 2 } \kappa _ { 4 } ( 1 + \kappa _ { 5 } ( \epsilon M / \kappa _ { 4 } ) ^ { - M / 4 } ) \tau ) } \left(\kappa _ { 6 } ^ { - 3 M / 4 } ( \epsilon M ) ^ { - 1 - M / 4 } ( h - \tau ) \left(-\epsilon^{3} M^{2}\left(\kappa_{4}^{M / 4} \kappa_{5}\right.\right.\right.\right. \\
& \left.\times\left(\kappa_{6}^{3 M / 4}+\kappa_{5}^{2}(\epsilon M)^{3 M / 4}\right)-\kappa_{5}\left(\kappa_{6}^{M / 2}\left(\epsilon \kappa_{4} M\right)^{M / 4}+\kappa_{5}^{2}\left(\epsilon^{2} \kappa_{4} \kappa_{6} M^{2}\right)^{M / 4}\right)\right) \\
& +16 b^{3} \kappa_{6}^{3 M / 4}(h-\tau) \tau\left(\kappa_{4}^{M / 4}(h-2 \tau)+(\epsilon M)^{M / 4}(-h+\tau)\right) \\
& +b \epsilon^{2} M\left(-h \kappa_{6}^{3 M / 4} M(\epsilon M)^{M / 4}+8 \kappa_{5} \kappa_{6}^{M / 2}\left(\epsilon \kappa_{4} M\right)^{M / 4} \tau+4 \kappa_{5}^{3}\left(\epsilon^{2} \kappa_{4} \kappa_{6} M^{2}\right)^{M / 4} \tau\right. \\
& \left.+\kappa_{4}^{M / 4} \kappa_{5}\left(\kappa_{6}^{3 M / 4}(h M-4 \tau)-8 \kappa_{5}^{2}(\epsilon M)^{3 M / 4} \tau\right)\right)+4 b^{2} \epsilon\left(-4 \kappa_{4}^{M / 4} \kappa_{5}^{3}(\epsilon M)^{3 M / 4} \tau^{2}\right. \\
& +4 \kappa_{5} \kappa_{6}^{M / 2}\left(\epsilon \kappa_{4} M\right)^{M / 4} \tau^{2}+\kappa_{6}^{3 M / 4}\left(h \kappa_{4}^{M / 4} M\right. \\
& \left.\left.\left.\left.\left.\times\left(h+\left(-2+\kappa_{5}\right) \tau\right)+M(\epsilon M)^{M / 4}\left(-h^{2}+\tau^{2}\right)\right)\right)\right)\right)\right),
\end{aligned}
$$

where $\kappa_{4}=\epsilon M+4 b h-4 b \tau, \kappa_{5}=(1+4 b \tau /(\epsilon M))^{M / 4}$ and $\kappa_{6}=\epsilon M+4 b \tau$. In the uniform case, $\tau=h / 2$ and thus the expression (22) considerably simplifies to the following expression:

$$
\int_{x_{i-1}}^{x_{i+1}} G^{i} \mathrm{~d} x=\left(\frac{h\left(-b h+\epsilon M\left(-1+(1+2 \rho / M)^{M / 2}\right)\right)}{b \epsilon\left(1+(1+2 \rho / M)^{M / 2}\right) M}\right)
$$


from which we get the desired result

$$
\lim _{M \rightarrow \infty} \int_{x_{i-1}}^{x_{i+1}} G^{i} \mathrm{~d} x=\frac{h}{b} \frac{e^{\rho}-1}{e^{\rho}+1} .
$$

COROLlaRY 3.4 If $\rho_{i}=b_{i} h / \epsilon$ is fixed, then the coefficients $\sigma_{i}, \eta_{i}$ and $\theta_{i}$ in Equation (12) converge to the coefficients of the numerical method (11). That is,

$$
\begin{aligned}
& \lim _{M \rightarrow \infty} \sigma_{i}\left(\epsilon, b_{i}, h, M\right)=B\left(\rho_{i}\right), \\
& \lim _{M \rightarrow \infty} \eta_{i}\left(\epsilon, b_{i}, h, M\right)=1, \\
& \lim _{M \rightarrow \infty} \theta_{i}\left(\epsilon, b_{i}, h, M\right)=1 .
\end{aligned}
$$

Proof Recall the definition of $\sigma_{i}$ from Equation (13), and use Lemmas 3.2 and 3.3, to get

$$
\begin{aligned}
\lim _{M \rightarrow \infty} \sigma_{i} & =\lim _{M \rightarrow \infty} \frac{h^{2}}{\epsilon} \frac{T_{2}}{T_{3}}=\frac{h^{2}}{\epsilon} \frac{\lim _{M \rightarrow \infty} T_{2}}{\lim _{M \rightarrow \infty} T_{3}}=\frac{h^{2}}{\epsilon} \frac{1 /\left(e^{\rho_{i}}+1\right)}{(h / b)\left(\left(e^{\rho_{i}}-1\right) /\left(e^{\rho_{i}}+1\right)\right)} \\
& =\rho_{i} \frac{1}{e^{\rho_{i}}-1}=B\left(\rho_{i}\right) .
\end{aligned}
$$

The proofs of Equations (25) and (26) are similar but also use Lemma 3.1.

THEOREM 3.5 The solution of the difference equation (12) converges, in the discrete maximum norm, to the exact solution of the problem (1) uniformly in $\epsilon$.

Proof This is a direct consequence of Corollary 3.4, together with Theorem 2 from Farrell [1].

\section{Computational results and discussion}

To examine the performance of the method, we carry out some numerical experiments. We first consider the following test problem:

$$
-\epsilon u^{\prime \prime}+u^{\prime}=2 x \quad \text { in }(0,1) \text { with } u(0)=u(1)=0 .
$$

We recall that the Green function associated with each global node is approximated on a Shishkin mesh in a pair of subintervals and the number of nodes used in those subintervals is denoted by $M$. Further recall that the number of nodes used in the global mesh is represented by $N$. We test the numerical method for several values of $N, M$ and $\epsilon$. We take $\epsilon=1, \epsilon=10^{-2}$ and $\epsilon=10^{-6}$, respectively, and for each of these $\epsilon$ values, we run the scheme for $N=2^{n}, 4 \leq n \leq 15$ and $M=2^{m}, 4 \leq m \leq 8$. We compute the error in the discrete maximum norm and compare the results with the exact scheme (11) (Tables 1-3). It is obvious that the numerical approximations obtained through the present method converge to the one obtained through the exact scheme as both local and global mesh sizes are refined.

Although the theory was not developed for internal layers, the method has the potential to catch them. We consider the following test problem to display that

$$
-\epsilon u^{\prime \prime}-2(2 x-1) u^{\prime}=4(2 x-1) \quad \text { in }(0,1) \text { with } u(0)=u(1)=0 .
$$

This problem has an internal layer at $x=0.5$. We report the corresponding numerical result in Table 4. 
Table 1. The error in discrete maximum norm for increasing values of $N$ and $M(\epsilon=1)$.

\begin{tabular}{|c|c|c|c|c|c|c|}
\hline \multirow[b]{2}{*}{$N \downarrow$} & \multicolumn{5}{|c|}{ Number of local mesh points $(M)$} & \multirow[b]{2}{*}{ Il'in } \\
\hline & 16 & 32 & 64 & 128 & 256 & \\
\hline $2^{4}$ & $1.2122 \mathrm{e}-004$ & $9.9112 \mathrm{e}-005$ & $8.9119 \mathrm{e}-005$ & $8.4424 \mathrm{e}-005$ & $8.2073 \mathrm{e}-005$ & $8.0122 \mathrm{e}-005$ \\
\hline $2^{5}$ & $4.2366 \mathrm{e}-005$ & $3.0481 \mathrm{e}-005$ & $2.4882 \mathrm{e}-005$ & $2.2311 \mathrm{e}-005$ & $2.1122 \mathrm{e}-005$ & $2.0060 \mathrm{e}-005$ \\
\hline $2^{6}$ & $1.6759 \mathrm{e}-005$ & $1.0623 \mathrm{e}-005$ & $7.6372 \mathrm{e}-006$ & $6.2271 \mathrm{e}-006$ & $5.5802 \mathrm{e}-006$ & $5.0165 \mathrm{e}-006$ \\
\hline $2^{7}$ & $7.2976 \mathrm{e}-006$ & $4.1943 \mathrm{e}-006$ & $2.6575 \mathrm{e}-006$ & $1.9099 \mathrm{e}-006$ & $1.5572 \mathrm{e}-006$ & $1.2543 \mathrm{e}-006$ \\
\hline $2^{8}$ & $3.3832 \mathrm{e}-006$ & $1.8255 \mathrm{e}-006$ & $1.0489 \mathrm{e}-006$ & $6.6450 \mathrm{e}-007$ & $4.7752 \mathrm{e}-007$ & $3.1357 \mathrm{e}-007$ \\
\hline $2^{9}$ & $1.6258 \mathrm{e}-006$ & $8.4602 \mathrm{e}-007$ & $4.5645 \mathrm{e}-007$ & $2.6225 \mathrm{e}-007$ & $1.6613 \mathrm{e}-007$ & $7.8393 \mathrm{e}-008$ \\
\hline $2^{10}$ & $7.9651 \mathrm{e}-007$ & $4.0649 \mathrm{e}-007$ & $2.1152 \mathrm{e}-007$ & $1.1411 \mathrm{e}-007$ & $6.5563 \mathrm{e}-008$ & $1.9599 \mathrm{e}-008$ \\
\hline $2^{11}$ & $3.9417 \mathrm{e}-007$ & $1.9913 \mathrm{e}-007$ & $1.0163 \mathrm{e}-007$ & $5.2884 \mathrm{e}-008$ & $2.8522 \mathrm{e}-008$ & $4.9020 \mathrm{e}-009$ \\
\hline $2^{12}$ & $1.9609 \mathrm{e}-007$ & $9.8541 \mathrm{e}-008$ & $4.9814 \mathrm{e}-008$ & $2.5376 \mathrm{e}-008$ & $1.3198 \mathrm{e}-008$ & $1.2191 \mathrm{e}-009$ \\
\hline $2^{13}$ & $9.7669 \mathrm{e}-008$ & $4.8885 \mathrm{e}-008$ & $2.4662 \mathrm{e}-008$ & $1.2467 \mathrm{e}-008$ & $6.3709 e-009$ & $3.5890 \mathrm{e}-010$ \\
\hline $2^{14}$ & $4.8865 \mathrm{e}-008$ & $2.4206 e-008$ & $1.2010 \mathrm{e}-008$ & $6.2972 \mathrm{e}-009$ & $3.2516 \mathrm{e}-009$ & $2.8985 \mathrm{e}-010$ \\
\hline $2^{15}$ & $2.5499 \mathrm{e}-008$ & $1.2208 \mathrm{e}-008$ & $5.7307 \mathrm{e}-009$ & $1.5420 \mathrm{e}-009$ & $1.4740 \mathrm{e}-011$ & $1.3413 \mathrm{e}-011$ \\
\hline
\end{tabular}

Table 2. The error in discrete maximum norm for increasing values of $N$ and $M\left(\epsilon=10^{-2}\right)$.

\begin{tabular}{|c|c|c|c|c|c|c|}
\hline \multirow[b]{2}{*}{$N \downarrow$} & \multicolumn{5}{|c|}{ Number of local mesh points $(M)$} & \multirow[b]{2}{*}{ Il'in } \\
\hline & 16 & 32 & 64 & 128 & 256 & \\
\hline $2^{4}$ & $5.2901 \mathrm{e}-002$ & $4.3039 \mathrm{e}-002$ & $4.1271 \mathrm{e}-002$ & $4.0581 \mathrm{e}-002$ & $4.0273 \mathrm{e}-002$ & $3.9988 \mathrm{e}-002$ \\
\hline $2^{5}$ & $6.3343 \mathrm{e}-002$ & $3.7863 \mathrm{e}-002$ & $2.5403 \mathrm{e}-002$ & $1.9269 \mathrm{e}-002$ & $1.6231 \mathrm{e}-002$ & $1.3212 \mathrm{e}-002$ \\
\hline $2^{6}$ & $6.3108 \mathrm{e}-002$ & $3.3637 \mathrm{e}-002$ & $1.8713 \mathrm{e}-002$ & $1.1202 \mathrm{e}-002$ & $7.4354 \mathrm{e}-003$ & $3.6931 \mathrm{e}-003$ \\
\hline $2^{7}$ & $4.0253 \mathrm{e}-002$ & $2.0741 \mathrm{e}-002$ & $1.0883 \mathrm{e}-002$ & $5.9254 \mathrm{e}-003$ & $3.4391 \mathrm{e}-003$ & $9.5057 \mathrm{e}-004$ \\
\hline $2^{8}$ & $2.1571 \mathrm{e}-002$ & $1.0950 \mathrm{e}-002$ & $5.6057 \mathrm{e}-003$ & $2.9248 \mathrm{e}-003$ & $1.5822 \mathrm{e}-003$ & $2.3944 \mathrm{e}-004$ \\
\hline $2^{9}$ & $1.0976 \mathrm{e}-002$ & $5.5300 \mathrm{e}-003$ & $2.7980 \mathrm{e}-003$ & $1.4296 \mathrm{e}-003$ & $7.4486 \mathrm{e}-004$ & $5.9974 \mathrm{e}-005$ \\
\hline $2^{10}$ & $5.5115 \mathrm{e}-003$ & $2.7663 e-003$ & $1.3914 \mathrm{e}-003$ & $7.0335 \mathrm{e}-004$ & $3.5919 \mathrm{e}-004$ & $1.5001 \mathrm{e}-005$ \\
\hline $2^{11}$ & $2.7584 \mathrm{e}-003$ & $1.3819 \mathrm{e}-003$ & $6.9299 \mathrm{e}-004$ & $3.4841 \mathrm{e}-004$ & $1.7608 \mathrm{e}-004$ & $3.7508 \mathrm{e}-006$ \\
\hline $2^{12}$ & $1.3795 \mathrm{e}-003$ & $6.9039 e-004$ & $3.4571 \mathrm{e}-004$ & $1.7334 \mathrm{e}-004$ & $8.7137 \mathrm{e}-005$ & $9.3772 \mathrm{e}-007$ \\
\hline $2^{13}$ & $6.8975 \mathrm{e}-004$ & $3.4504 \mathrm{e}-004$ & $1.7265 \mathrm{e}-004$ & $8.6444 \mathrm{e}-005$ & $4.3339 \mathrm{e}-005$ & $2.3442 \mathrm{e}-007$ \\
\hline $2^{14}$ & $3.4487 \mathrm{e}-004$ & $1.7248 \mathrm{e}-004$ & $8.6270 \mathrm{e}-005$ & $4.3165 \mathrm{e}-005$ & $2.1612 \mathrm{e}-005$ & $5.8556 \mathrm{e}-008$ \\
\hline $2^{15}$ & $1.7243 \mathrm{e}-004$ & $8.6227 \mathrm{e}-005$ & $4.3121 \mathrm{e}-005$ & $2.1568 \mathrm{e}-005$ & $1.0791 \mathrm{e}-005$ & $1.3648 \mathrm{e}-008$ \\
\hline
\end{tabular}

Table 3. The error in discrete maximum norm for increasing values of $N$ and $M\left(\epsilon=10^{-6}\right)$.

\begin{tabular}{lcccccc}
\hline \multicolumn{5}{c}{ Number of local mesh points $(M)$} & \\
\cline { 2 - 6 }$N \downarrow$ & 16 & 32 & 64 & 128 & 256 & Il'in \\
\hline $2^{4}$ & $5.8592 \mathrm{e}-002$ & $5.8592 \mathrm{e}-002$ & $5.8592 \mathrm{e}-002$ & $5.8592 \mathrm{e}-002$ & $5.8592 \mathrm{e}-002$ & $5.8592 \mathrm{e}-002$ \\
$2^{5}$ & $3.0272 \mathrm{e}-002$ & $3.0272 \mathrm{e}-002$ & $3.0272 \mathrm{e}-002$ & $3.0272 \mathrm{e}-002$ & $3.0272 \mathrm{e}-002$ & $3.0272 \mathrm{e}-002$ \\
$2^{6}$ & $1.5379 \mathrm{e}-002$ & $1.5379 \mathrm{e}-002$ & $1.5379 \mathrm{e}-002$ & $1.5379 \mathrm{e}-002$ & $1.5379 \mathrm{e}-002$ & $1.5379 \mathrm{e}-002$ \\
$2^{7}$ & $7.7495 \mathrm{e}-003$ & $7.7495 \mathrm{e}-003$ & $7.7495 \mathrm{e}-003$ & $7.7495 \mathrm{e}-003$ & $7.7495 \mathrm{e}-003$ & $7.7495 \mathrm{e}-003$ \\
$2^{8}$ & $3.8890 \mathrm{e}-003$ & $3.8890 \mathrm{e}-003$ & $3.8890 \mathrm{e}-003$ & $3.8890 \mathrm{e}-003$ & $3.8890 \mathrm{e}-003$ & $3.8890 \mathrm{e}-003$ \\
$2^{9}$ & $1.9473 \mathrm{e}-003$ & $1.9473 \mathrm{e}-003$ & $1.9473 \mathrm{e}-003$ & $1.9473 \mathrm{e}-003$ & $1.9473 \mathrm{e}-003$ & $1.9473 \mathrm{e}-003$ \\
$2^{10}$ & $9.7361 \mathrm{e}-004$ & $9.7361 \mathrm{e}-004$ & $9.7361 \mathrm{e}-004$ & $9.7361 \mathrm{e}-004$ & $9.7361 \mathrm{e}-004$ & $9.7361 \mathrm{e}-004$ \\
$2^{11}$ & $4.8604 \mathrm{e}-004$ & $4.8604 \mathrm{e}-004$ & $4.8604 \mathrm{e}-004$ & $4.8604 \mathrm{e}-004$ & $4.8604 \mathrm{e}-004$ & $4.8604 \mathrm{e}-004$ \\
$2^{12}$ & $2.4209 \mathrm{e}-004$ & $2.4208 \mathrm{e}-004$ & $2.4208 \mathrm{e}-004$ & $2.4208 \mathrm{e}-004$ & $2.4208 \mathrm{e}-004$ & $2.4208 \mathrm{e}-004$ \\
$2^{13}$ & $1.2045 \mathrm{e}-004$ & $1.2006 \mathrm{e}-004$ & $1.2006 \mathrm{e}-004$ & $1.2006 \mathrm{e}-004$ & $1.2006 \mathrm{e}-004$ & $1.2006 \mathrm{e}-004$ \\
$2^{14}$ & $6.5135 \mathrm{e}-005$ & $1.1517 \mathrm{e}-004$ & $5.9032 \mathrm{e}-005$ & $5.9032 \mathrm{e}-005$ & $5.9032 \mathrm{e}-005$ & $5.9032 \mathrm{e}-005$ \\
$2^{15}$ & $1.1517 \mathrm{e}-004$ & $2.9680 \mathrm{e}-005$ & $2.8527 \mathrm{e}-005$ & $2.8517 \mathrm{e}-005$ & $2.8517 \mathrm{e}-005$ & $2.8517 \mathrm{e}-005$ \\
\hline
\end{tabular}


Table 4. The error in discrete maximum norm for increasing values of $N$ and $M\left(\epsilon=10^{-2}\right)$.

\begin{tabular}{lcccccc}
\hline \multicolumn{7}{c}{ Number of local mesh points $(M)$} \\
\cline { 2 - 6 }$N \downarrow$ & 16 & 32 & 64 & 128 & 256 & Il'in \\
\hline $2^{4}$ & $3.4150 \mathrm{e}-001$ & $3.5784 \mathrm{e}-001$ & $2.8359 \mathrm{e}-001$ & $2.5883 \mathrm{e}-001$ & $2.5778 \mathrm{e}-001$ & $2.6892 \mathrm{e}-001$ \\
$2^{5}$ & $2.0838 \mathrm{e}-001$ & $1.2350 \mathrm{e}-001$ & $1.0858 \mathrm{e}-001$ & $1.2002 \mathrm{e}-001$ & $1.2575 \mathrm{e}-001$ & $1.3150 \mathrm{e}-001$ \\
$2^{6}$ & $1.1664 \mathrm{e}-001$ & $6.8098 \mathrm{e}-002$ & $5.4364 \mathrm{e}-002$ & $5.9475 \mathrm{e}-002$ & $6.2068 \mathrm{e}-002$ & $6.4685 \mathrm{e}-002$ \\
$2^{7}$ & $7.7242 \mathrm{e}-002$ & $4.3710 \mathrm{e}-002$ & $2.7433 \mathrm{e}-002$ & $2.9507 \mathrm{e}-002$ & $3.0747 \mathrm{e}-002$ & $3.2150 \mathrm{e}-002$ \\
$2^{8}$ & $6.0824 \mathrm{e}-002$ & $3.2896 \mathrm{e}-002$ & $1.8772 \mathrm{e}-002$ & $1.4807 \mathrm{e}-002$ & $1.5288 \mathrm{e}-002$ & $1.6048 \mathrm{e}-002$ \\
$2^{9}$ & $5.3586 \mathrm{e}-002$ & $2.7966 \mathrm{e}-002$ & $1.5097 \mathrm{e}-002$ & $8.6818 \mathrm{e}-003$ & $7.6768 \mathrm{e}-003$ & $8.0101 \mathrm{e}-003$ \\
$2^{10}$ & $5.0258 \mathrm{e}-002$ & $2.5677 \mathrm{e}-002$ & $1.3359 \mathrm{e}-002$ & $7.2197 \mathrm{e}-003$ & $4.1727 \mathrm{e}-003$ & $4.0005 \mathrm{e}-003$ \\
$2^{11}$ & $4.8680 \mathrm{e}-002$ & $2.4598 \mathrm{e}-002$ & $1.2540 \mathrm{e}-002$ & $6.5213 \mathrm{e}-003$ & $3.5287 \mathrm{e}-003$ & $1.9991 \mathrm{e}-003$ \\
\hline
\end{tabular}

\section{Conclusion}

We consider an $\epsilon$-uniform numerical method for a singularly perturbed two-point BVP, whose significance is that, although it uses an equidistant mesh, it requires no exact solution of a local BVP. The resulting numerical method can also be proved to converge to the true solution of the BVP uniformly in $\epsilon$, which shows that it is possible to develop a fully discrete $\epsilon$-uniform numerical method on uniform meshes. We also note that the method has the potential to catch internal layers. Finally, we report that the numerical experiments are in good agreement with the theoretical results.

\section{References}

[1] P.A. Farrell, Sufficient conditions for the uniform convergence of difference schemes for singularly perturbed turning and non-turning point problems, in Computational and Asymptotic Methods for Boundary and Interior Layers, J.J.H. Miller, ed., Boole Press, Dublin, 1982, pp. 230-235.

[2] A.M. Il'in, Differencing scheme for a partial differential equation with a small parameter affecting the highest derivative, Math. Notes 6 (1969), pp. 596-602.

[3] T. Linß, Finite difference schemes for convection diffusion problems with a concentrated source and a discontinuous convection field, Comput. Methods Appl. Math. 2(1) (2002), pp. 41-49.

[4] J. Miller, E. O'Riordan, and G. Shishkin, Fitted Numerical Methods for Singularly Perturbed Problems, World Scientific, Singapore, 1996.

[5] H.-G. Roos, M. Stynes, and L. Tobiska, Numerical Methods for Singularly Perturbed Differential Equations, Springer-Verlag, Berlin, 1996.

[6] G.I. Shishkin, A difference scheme for singularly perturbed equation of parabolic type with a discontinuous initial condition, Soviet Math. Dokl. 37 (1988), pp. 792-796.

[7] G.I. Shishkin, Grid approximation of singularly perturbed elliptic and parabolic equations, Second Doctoral thesis, Keldysh Institute, Moscow, 1990 (in Russian). 